\title{
Octadecyl Carbon Chain-Bonded Silica
}

National Cancer Institute

\section{Source}

National Cancer Institute. Octadecyl Carbon Chain-Bonded Silica. NCI Thesaurus. Code C161776.

Silica beads that are bound to octadecyl carbon chains and are used to fill columns as the stationary phase for a number of chromatography-based techniques. 